\title{
Highly Stretchable and Highly Resilient Polymer-Clay Nanocomposite Hydrogels with Low Hysteresis
}

\author{
Xing Su, ${ }^{1}$ Suntharavathanan Mahalingam, ${ }^{2}$ Mohan Edirisinghe, ${ }^{2}$ Biqiong Chen ${ }^{1, *}$ \\ *Corresponding author. Email: biqiong.chen@sheffield.ac.uk.
}

\begin{abstract}
${ }^{1}$ Department of Materials Science and Engineering, University of Sheffield, Mappin Street, Sheffield S1 3JD, United Kingdom.
\end{abstract}

${ }^{2}$ Department of Mechanical Engineering, University College London, Torrington Place, London WC1E 7JE, United Kingdom.

\begin{abstract}
Highly stretchable and highly resilient polymer-clay nanocomposite hydrogels were synthesized by in situ polymerization of acrylamide in the presence of pristine montmorillonite (MMT) or chitosan-treated MMT nanoplatelets at an elevated temperature. Both nanocomposite hydrogels can be stretched to a strain of no less than $1290 \%$. The treatment of clay with chitosan improves the tensile strength, elongation at break and energy at break of the nanocomposite hydrogel by $237 \%, 102 \%$ and $389 \%$, respectively, due to the strong chitosan-MMT electrostatic interaction and the grafting of polyacrylamide onto chitosan chains. Both hydrogels display excellent resilience with low hysteresis; with a maximum tensile strain of $50 \%$ ultralow hysteresis is found, while with a maximum strain of $500 \%$ both hydrogels fully recover their original state in just 1 minute. The superb resilience of the nanocomposite hydrogels is attributed to the strong interactions within the hydrogels brought by chain branching, multiple hydrogen
\end{abstract}


bonding, covalent bonding and/or electrostatic force. The hydrogels can be fabricated into different shapes and forms, including microfibers spun using pressurized gyration, which may find a variety of potential applications in particular in healthcare.

KEYWORDS: nanocomposite hydrogel, montmorillonite, structure, mechanical properties, elasticity, hysteresis

\section{INTRODUCTION}

Hydrogels are a class of polymeric, hydrophilic materials which are able to absorb and keep water in their crosslinked network. ${ }^{1}$ They may be fabricated into different forms including microgels, ${ }^{2-4}$ and used as agricultural products,${ }^{5}$ food ingredients ${ }^{6}$ and biomaterials such as tissue scaffolds, ${ }^{7}$ drug delivery systems,${ }^{8}$ wound dressings ${ }^{9}$ and biosensors. ${ }^{10}$ However, conventional chemically crosslinked hydrogels are often weak and/or brittle, which limits hydrogels from wider applications. $^{11}$

To overcome this shortcoming, polymer-clay nanocomposite hydrogels have attracted much attention in the last decade for synthesizing tough hydrogels because highly functionalized clay can form physical, reversible and dense crosslinks which are effective on dissipating energy ${ }^{2}$ and stabilizing the network. ${ }^{11}$ Haraguchi et al. ${ }^{11,12}$ developed a series of poly(N-isopropyl acrylamide)synthetic hectorite nanocomposite hydrogels. Unlike conventional chemically crosslinked hydrogels, these nanocomposite hydrogels could reach a tensile strength of $109 \mathrm{kPa}$ and a strain at break over $800 \%$. The ionic interaction between initiator molecules and clay surface was essential to hydrogel formation, which enables multiple polymer macromolecules to grow on clay surface. ${ }^{13}$ Polyacrylamide (PAM) was frequently used to synthesize nanocomposite hydrogels because of its 
superb hydrophilicity, flexibility, simplicity in synthesis, ${ }^{14}$ and biocompatibility. ${ }^{15}$ Montmorillonite (MMT), with the ideal chemical formula of $\mathrm{Al}_{2} \mathrm{Si}_{4} \mathrm{O}_{10}(\mathrm{OH})_{2} \cdot \mathrm{yH}_{2} \mathrm{O},{ }^{16}$ was often used as a complementary physical crosslinker of chemically crosslinked PAM hydrogels. ${ }^{17-20}$ MMT is also biocompatible and can be readily expelled from the body after metabolism and excretion. ${ }^{21}$ Until 2015, ultra-stretchable, self-healable and tough PAM-MMT physically crosslinked hydrogels were synthesized, which presented an extremely high strain at break of $12000 \% .{ }^{22}$ After stretched to a strain of $2000 \%$, a residual strain of $500 \%$ and a high hysteresis were observed after 1 minute, and a full shape recovery required 5 days' storage at $25{ }^{\circ} \mathrm{C} .{ }^{22}$ It is considered challenging to achieve low hysteresis with physically crosslinked PAM-clay hydrogels because of temporary or even permanent breaking of some organic-inorganic crosslinks. ${ }^{23-24}$

High hysteresis often means reduced mechanical properties of hydrogels after being cyclically loaded, so attempts were made to overcome hysteresis of hydrogels including well designed hydrogels composed of copolymerized tetra-armed monomers ${ }^{23-24}$ and physically crosslinked PAM-synthetic hectorite nanocomposite hydrogels. ${ }^{25}$ In the latter (with a starting weight ratio of $1: 1$ for the monomer:clay), a hysteresis of $19 \%$ in the first cyclic testing to a $1000 \%$ strain and a reduced hysteresis of $9 \%$ in later cycles were found. ${ }^{25}$ The high crosslinking density and hydrophilic, flexible nature of PAM gave rise to a small internal friction force and thus low hysteresis. ${ }^{25}$ These promising results indicate that it may be possible to achieve low hysteresis with PAM-MMT nanocomposite hydrogels.

Chitosan (CHI) is a biopolymer produced from chitin, which is rich in the crustacean and mollusk shells. ${ }^{26}$ Due to its excellent biocompatibility and biodegradability, chitosan is widely used in healthcare applications. ${ }^{27-29}$ It is positively charged in an acidic environment, which has 
potential of strongly bonding to the negatively charged MMT surface ${ }^{30-31}$ by electrostatic interactions. ${ }^{32-33}$

This work aimed to develop polymer-clay nanocomposite hydrogels with high extensibility, excellent resilience and low hysteresis. Unlike previous work on PAM-clay nanocomposites, ${ }^{11,22,25}$ in situ polymerization of acrylamide (AM) in the presence of MMT or chitosan-treated MMT (CHI-MMT) was conducted at a higher temperature of $60{ }^{\circ} \mathrm{C}$ without a catalyst, instead of room temperature with a catalyst, because it is favored for chain grafting and branching at this temperature. ${ }^{34}$ During graft polymerization, the radical initiator was expected to attack the hydroxyl groups of the polysaccharide chains to generate alkoxy radicals, which would also initiate the polymerization of AM. ${ }^{35}$ A chitosan grafted PAM-MMT hydrogel was recently synthesized by graft polymerization of $\mathrm{AM}$ onto chitosan at $60^{\circ} \mathrm{C}$ in the presence of MMT platelets and a chemical crosslinker N,N'-methylenebisacrylamide. ${ }^{19}$ However, the mechanical properties of the hydrogels were not reported. In the present study, no chemical crosslinker was used, and the mechanical properties of the nanocomposite hydrogels such as Young's modulus, tensile strength, strain at break, hysteresis ratio and rheological properties were investigated by tensile, cyclic tensile and rheological tests. Their results were interpreted in depth with the employment of the findings from X-ray diffraction (XRD), Fourier transform infared spectroscopy (FTIR), dynamic scanning calorimetry (DSC), scanning electron microscopy (SEM) and in vitro degradation tests. The mechanical properties of the fully swollen nanocomposite hydrogels were also investigated, which are often overlooked in the literature. To demonstrate the manufacturing versability of these hydrogels, various shapes of hydrogels were prepared, together with hydrogel microfibers.

\section{EXPERIMENTAL}


Materials. Acrylamide and potassium persulfate (KPS) with an assay level $\geq 99.0 \%$ were purchased from Sigma Aldrich. MMT with a cation exchange capability of 90 meq $(100 \mathrm{~g})^{-1}$ was supplied by Southern Clay Products. Chitosan with an average molecular weight from 100,000 to 300,000 was provided by Acros Organics. Acetic acid with a trade name of TraceSELECT® Ultra, purity $\geq 99.0 \%, \mathrm{~N}, \mathrm{~N}, \mathrm{~N}^{\prime}, \mathrm{N}^{\prime}$-tetramethylethylenediamine (TEMED), purity $\geq 99.0 \%$, and phosphate buffer saline (PBS) tablets and lysozyme lyophilized powder with protein content $\geq 90 \%(\geq 40,000$ unit $\mathrm{mg}^{-1}$ ) were purchased from Sigma Aldrich. All chemicals were used as received.

Preparation of hydrogels. $15 \mathrm{~g}$ MMT was dispersed in $1000 \mathrm{ml}$ distilled water for 24 hours on a table roller, followed by a 30 mins' sonication using a probe sonicator with a frequency of 5 $\mathrm{Hz}$ and another 24 hours' standstill settling in order to eliminate the un-exfoliated clay platelets and impurities. The precipitate was dried and weighed and the exfoliated MMT left in the supernatant was collected and its concentration in water was determined to be $0.012 \mathrm{~g} \mathrm{~L}^{-1}$.

The typical process of preparing PAM/CHI MMT hydrogels, denoted as PM5CHI, was described below, which was inspired by the methods of Gao et al. ${ }^{22}$ and Ferfera et al. ${ }^{19}$ for the synthesis of PAM-MMT nanocomposite hydrogels and a chitosan grafted PAM-MMT hydrogel, respectively. $21.5 \mathrm{ml}$ of the above MMT water suspension (containing $0.25 \mathrm{~g} \mathrm{MMT}$ ) was measured and sonicated for 10 minutes, to which $0.2 \mathrm{ml}$ acetic acid and $0.044 \mathrm{~g}$ chitosan were added. MMT content in the hydrogel was fixed at $0.89 \mathrm{wt} \%$ based on the results from previous work on PAMMMT nanocomposite hydrogels. ${ }^{22}$ Chitosan content was fixed at $0.16 \mathrm{wt} \%$ as a higher amount could cause direct gelation of the mixture and inhibit polymerization of AM. After 4 hours of stirring under $300 \mathrm{rpm}$, the suspension was sonicated for 10 minutes. Then the KPS solution $(0.078$ $\mathrm{g}$ in $3.44 \mathrm{ml}$ distilled water) was added into the CHI-MMT suspension under vigorous stirring for 
10 minutes. The mixture was heated at $60{ }^{\circ} \mathrm{C}$ in a water bath for 10 minutes in order to generate free radicals. After the mixture was cooled to ambient temperature, $5 \mathrm{~g}$ AM was added, followed by 1 hour's stirring. Finally, the reaction mixture was transferred into plastic tubes and sealed. Polymerization was continued in a water bath at $60^{\circ} \mathrm{C}$ for 24 hours. The preparation of PAM MMT hydrogels, denoted as PM5, followed the same protocol as above, except that chitosan was not used. The weight ratios of MMT:AM and AM: $\mathrm{H}_{2} \mathrm{O}$ were fixed at 0.05 and 0.2 , respectively. ${ }^{22}$

AM was also polymerized in water with the presence of chitosan but without MMT, denoted as PAMCHI, and in the absence of both CHI and MMT, denoted as PAM. In addition, PAM MMT nanocomposite hydrogels were prepared following Gao et al's method ${ }^{22}$ at $25^{\circ} \mathrm{C}$ with $\mathrm{N}, \mathrm{N}, \mathrm{N}^{\prime}, \mathrm{N}^{\prime}-$ tetramethylethylenediamine as the catalyst, denoted as PM5cata. These materials were prepared as control samples for comparison.

Characterization. The tensile tests were conducted on a Hounsfield universal mechanical testing machine using a $10 \mathrm{~N}$ load cell and a crosshead speed of $100 \mathrm{~mm} \mathrm{~min}^{-1}$. The test samples were rod-like with $3 \mathrm{~mm}$ diameter, $15 \mathrm{~mm}$ full length and $5 \mathrm{~mm}$ gauge length. For each material, 20 samples were tested. For cyclic tensile tests, each sample was subject to 8-cycle tension. Different maximum strains of 50\%, 300\% and 500\% were studied. Five samples were tested for each material. The hysteresis ratio $h$ for a certain cycle was calculated by the equation: $h=1$ $\left(\mathrm{A}_{2}\right) /\left(\mathrm{A}_{1}\right)$, where $\mathrm{A}_{1}$ and $\mathrm{A}_{2}$ represent the areas beneath the tensile stress-strain curve and the return curve, respectively. ${ }^{36}$

X-Ray Diffraction was performed on a STOE STADI P $(\mathrm{Cu} K \alpha)$ Diffractometer. The transmission mode was selected with an incident X-ray wavelength of $0.154 \mathrm{~nm}$ at a scan rate of $1.0^{\circ} \mathrm{min}^{-1}$. Fourier transform infrared spectroscopy was conducted on a Spectrum 100 
spectrophotometer (PerkinElmer) in the wavenumber region from 4000 to $600 \mathrm{~cm}^{-1}$ with a resolution of $1 \mathrm{~cm}^{-1}$ using the hydrogels dehydrated in a vacuum oven at $50{ }^{\circ} \mathrm{C}$ overnight. Dynamic scanning calorimetry was carried out on a PERKIN ELMER DSC 6 with the temperature ranging from 25 to $250{ }^{\circ} \mathrm{C}$ at a rate of $10{ }^{\circ} \mathrm{C} \mathrm{min}-1$ under a nitrogen flow rate of $20 \mathrm{ml} \mathrm{min}^{-1}$. The second heating curve was used for analysis. Dehydrated hydrogel samples were ground into powder for both XRD and DSC.

In vitro degradation tests were carried out following the method described in the literature. ${ }^{37,38}$ Briefly, the cylindrical hydrogel rods with a thickness of $5 \mathrm{~mm}$ were immersed in PBS solution $(\mathrm{pH}=7.4)$ at $37{ }^{\circ} \mathrm{C}$ containing lysozyme at a concentration of $1.5 \mu \mathrm{g} \mathrm{ml}^{-1}$, simulating the internal environment of human body. ${ }^{39,40}$ After a pre-determined period of time, samples were removed from the solution, washed with distilled water, dried in the vacuum oven at $40{ }^{\circ} \mathrm{C}$ and weighed. The buffer solution was changed every 24 hours in order to maintain the activity of the enzyme. Five specimens of each material were tested. Scanning electron microscopy was utilised to observe the morphology of the cross-sectional surface of the freeze-dried nanocomposite hydrogels with carbon coating. Samples were imaged using an Inspect F FEG SEM operated at a voltage of $5 \mathrm{kV}$. Samples for swelling tests were freeze-dried (FreeZone Triad Cascade Benchtop Freeze Dry System, Labconco Corporation) under vacuum at $-15^{\circ} \mathrm{C}$ for 2 days. Swelling tests were conducted by immersing the freeze-dried samples in distilled water at $25^{\circ} \mathrm{C}$ for a period of time to reach the equilibrium state. The equilibrium swelling ratios $\varphi$ were defined as $\varphi=\left(\mathrm{W}_{\mathrm{s}}-\mathrm{W}_{\mathrm{d}}\right) / \mathrm{W}_{\mathrm{d}} \times 100 \%$, where the weight of the fully swollen sample $\mathrm{W}_{\mathrm{s}}$ and the weight of the initial freeze-dried sample $\mathrm{W}_{\mathrm{d}}$ were determined using an analytical balance.

Rheological tests were conducted on an AR2000 Advanced Rheometer (TA Instruments). At 25 ${ }^{\circ} \mathrm{C}$, the storage moduli $\mathrm{G}$ ' and loss moduli $\mathrm{G}$ " of the PM5 and PM5CHI hydrogels were determined 
over an angular frequency range from 0.1 to $100 \mathrm{rad} \mathrm{s}^{-1}$ under a fixed strain of $1 \%$, which was in the linear viscoelastic region pre-determined by strain sweep tests. All tests were performed using a parallel-plate geometry with a diameter of $20 \mathrm{~mm}$ along with a solvent trap to avoid evaporation. The gap between two parallel plates was $1.0 \mathrm{~mm}$.

Fabrication of hydrogel fibers. Hydrogel fibers were fabricated, via the precursor route, using pressurized gyration. In this method centrifugal force and solution blowing were coupled to extrude the precursor fibers, following the procedure established in our previous work. ${ }^{41}$ The hydrogel precursor material was prepared by mixing water, MMT, chitosan, initiator and monomer using the same method and at the same ratio as for preparing the PM5CHI hydrogel, before being sealed in plastic tubes. It had been kept at ambient temperature for approximately 3 hours before being stored in a fridge until use. Around $1 \mathrm{ml}$ of the precursor material was dissolved in $10 \mathrm{ml}$ of deionised water under magnetic stirring for 10 minutes at ambient temperature. Thereafter, the solution was transferred to the gyration vessel and spun at $36000 \mathrm{rpm}$ rotating speed and $0.1 \mathrm{MPa}$ working pressure. SEM of the resultant fibers was performed using a JSM-6301F SEM. The micrographs were recorded at an operating voltage of $20 \mathrm{kV}$ and an emission current $6 \mu \mathrm{A}$ to obtain high resolution images.

\section{RESULTS AND DISCUSSION}

Formation of nanocomposite hydrogels. The synthesis of nanocomposite hydrogels is illustrated in Scheme 1. For PM5, MMT was exfoliated in distilled water by means of table rolling and sonication. Then KPS initiator was added and thus adsorbed onto the MMT platelets by ionic interaction shown in Scheme 2 (a). ${ }^{11}$ Finally, AM monomer was introduced, some of 


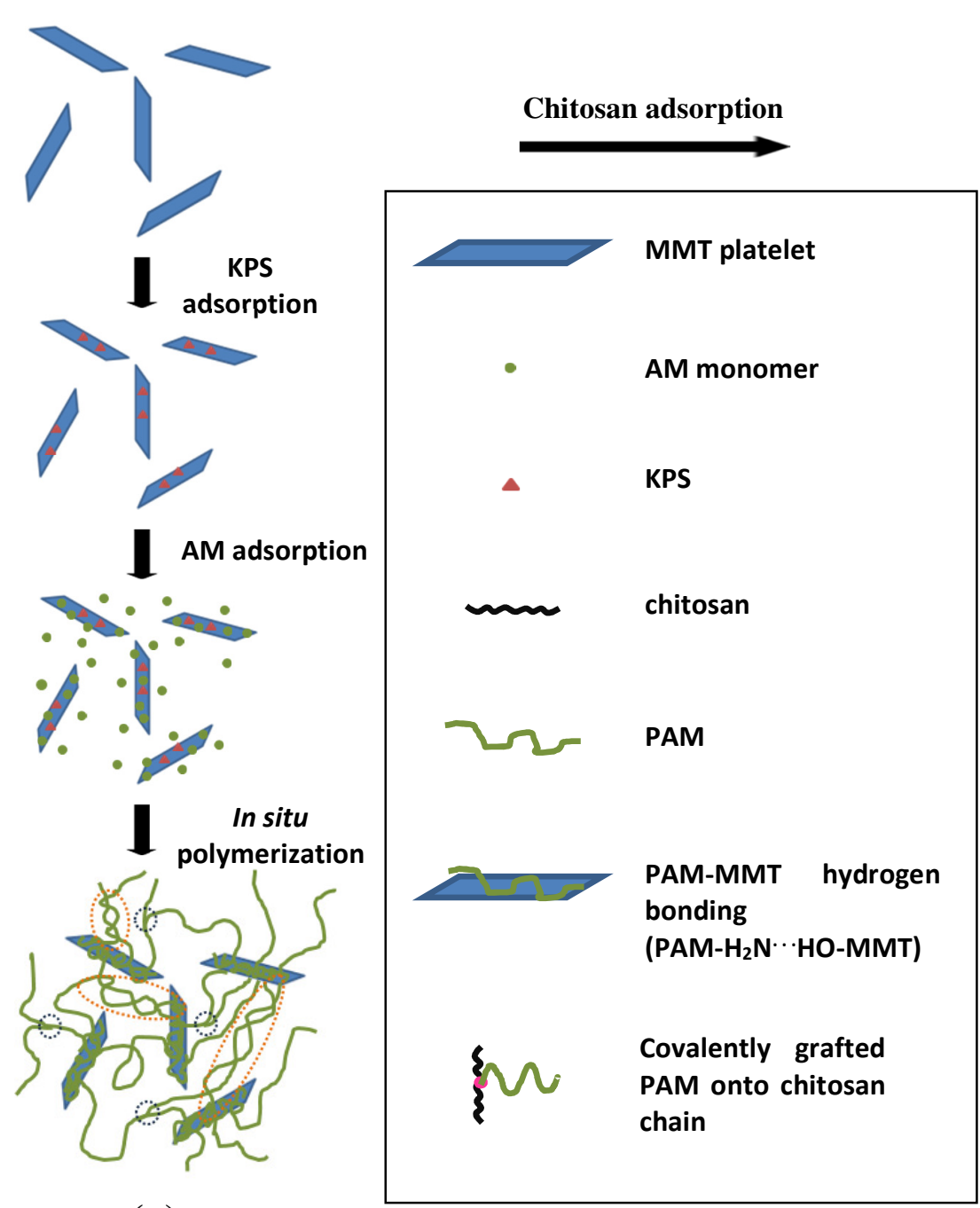

(a)

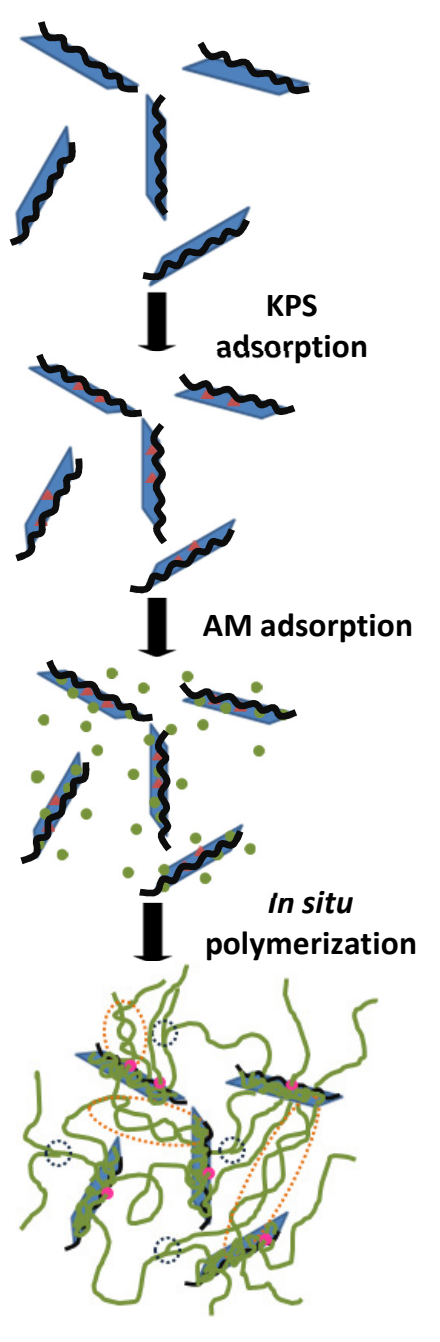

(b)

Scheme 1. Schematic illustration of the synthesis of (a) PM5 and (b) PM5CHI hydrogels. The brown and blue circles indicated PAM chain entanglement and PAM branching respectively. The pink dots in (b) represented the points where AM grafted onto chitosan chains.
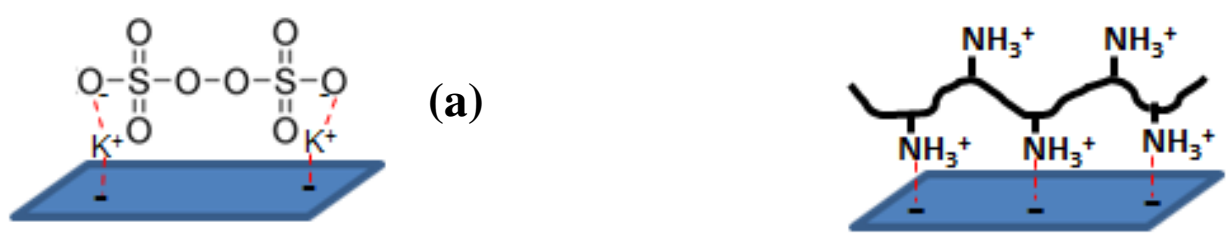

Scheme 2. Schematic illusion of (a) KPS-MMT ionic interaction, and (b) chitosan-MMT electrostatic interaction. 


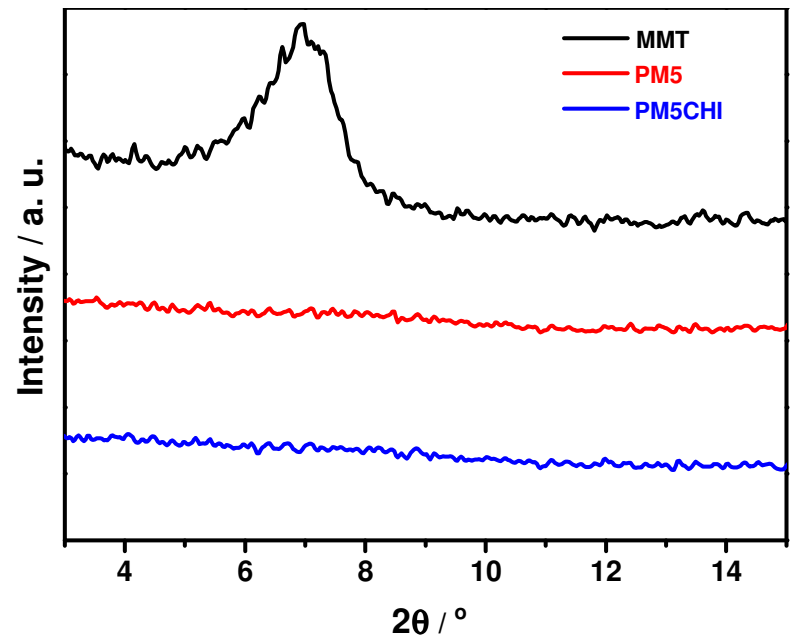

Figure 1. XRD spectra of the neat MMT and PM5 and PM5CHI dry samples.

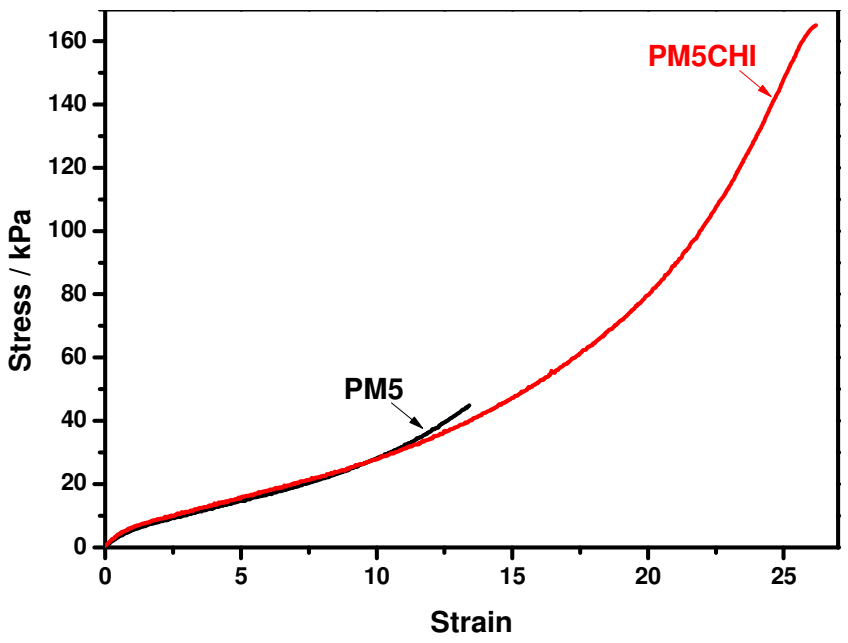

Figure 2. Representative tensile stress-strain curves of PM5 and PM5CHI hydrogels.

which was also adsorbed onto the MMT platelets, and polymerized. For PM5CHI, MMT was first combined with chitosan by electrostatic interaction shown in Scheme 2 (b). Then some of KPS attacked the hydroxyl groups of chitosan chains to form radicals. Both chitosan radicals and KPS initiated the in situ polymerization of AM. As seen from XRD traces (Figure 1), the neat MMT showed a strong peak at $2 \theta=7.1^{\circ}$, corresponding to an interlayer spacing of $1.23 \mathrm{~nm} .{ }^{42}$ However, this peak disappeared in both PM5 and PM5CHI, confirming that MMT platelets were well exfoliated in both nanocomposite hydrogels since the content of MMT in the dry nanocomposites was relatively high, i.e. 4.6 wt. $\%$. PAM chain entanglement, PAM branches and PAM-MMT hydrogen bonding were expected in both hydrogels, ${ }^{43}$ which is discussed subsequently. 
Mechanical properties of nanocomposite hydrogels. The nanocomposite hydrogels were tensile tested and their results are presented in Figure 2 and Table 1. The hydrogels with and without chitosan treatment were capable of achieving a high elongation at break of over $1000 \%$ without any sign of failure, similar to some previously reported nanocomposite hydrogels. ${ }^{12}$ PM5 and PM5CHI displayed nearly the same testing curve until the failure of PM5, suggesting the two hydrogels have similar macroscopic tensile behaviour up to a strain of over $1000 \%$ including the same Young's modulus. When stretched, the entangled PAM macromolecular chains were extended in both hydrogels and the clay platelets tended to orient parallel to the stretching direction. ${ }^{44}$ Upon continuous stretching, some of the hydrogen bonds formed between amide groups of PAM and silanol groups of MMT platelets started to break. As for PM5CHI, apart from the breakage of PAM-MMT hydrogen bonding, chitosan chains were also stretched and moved across the MMT surface. ${ }^{32}$

Table 1. Tensile properties of PM5 and PM5CHI hydrogels.

\begin{tabular}{llcll}
\hline Sample & $\begin{array}{l}\text { Young's modulus } \\
/ \mathrm{kPa}\end{array}$ & $\begin{array}{l}\text { Tensile strength } \\
/ \mathrm{kPa}\end{array}$ & Strain at break & $\begin{array}{l}\text { Energy at break } \\
/ \mathrm{MJ} \mathrm{m}^{-3}\end{array}$ \\
\hline PM5 & $7.1 \pm 0.3$ & $45.9 \pm 3.0$ & $12.9 \pm 0.7$ & $0.27 \pm 0.02$ \\
\hline PM5CHI & $7.3 \pm 0.3$ & $154.8 \pm 9.0$ & $26.0 \pm 0.6$ & $1.32 \pm 0.06$ \\
\hline
\end{tabular}

By comparing PM5 and PM5CHI, the latter presented about 240\% higher tensile strength, 100\% higher strain at break and 390\% higher energy at break, reaching a tensile strength of $154.8 \mathrm{kPa}$, a strain at break of 26.0 and a tensile energy at break of $1.32 \mathrm{MJ} \mathrm{m}^{-3}$ (Table 1). The high strength of the PM5CHI hydrogel suggested that load was transferred efficiently to chitosan-treated MMT platelets presumably through the covalent bonds between PAM chains and chitosan chains after the grafted PAM chains had been extended. ${ }^{45}$ The increase of tensile strength could also be 
attributed to the strong electrostatic interactions between chitosan and MMT, which was much stronger than the hydrogen bonding between PAM and MMT platelets and so would require higher stress to detach chitosan chains from the MMT platelets to reach the final failure. The increase of the strain at break in the PM5CHI hydrogel was due to the additional energy dissipation by unzipping the electrostatically bonded chitosan and MMT. ${ }^{32}$ Combining the enhanced tensile strength, strain at break and the same Young's modulus, a much higher energy at break was achieved by PM5CHI. The excellent flexibility and extensibility of both the hydrogels were demonstrated in Figure 3, where the hydrogels could be either knotted or stretched to a large deformation.

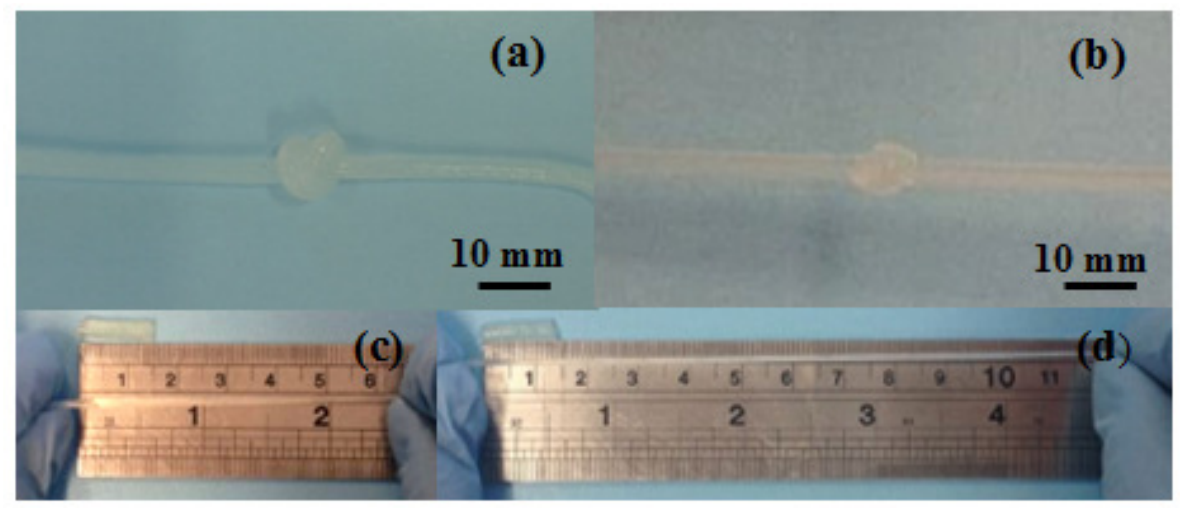

Figure 3. Photographs of PM5 and PM5CHI hydrogels, (a, b) knotted PM5 and PM5CHI, (c) PM5 with and without a tensile strain of 12, and (d) PM5CHI with and without a strain of 25. Only the middle part of the hydrogel rods above the ruler was stretched. 

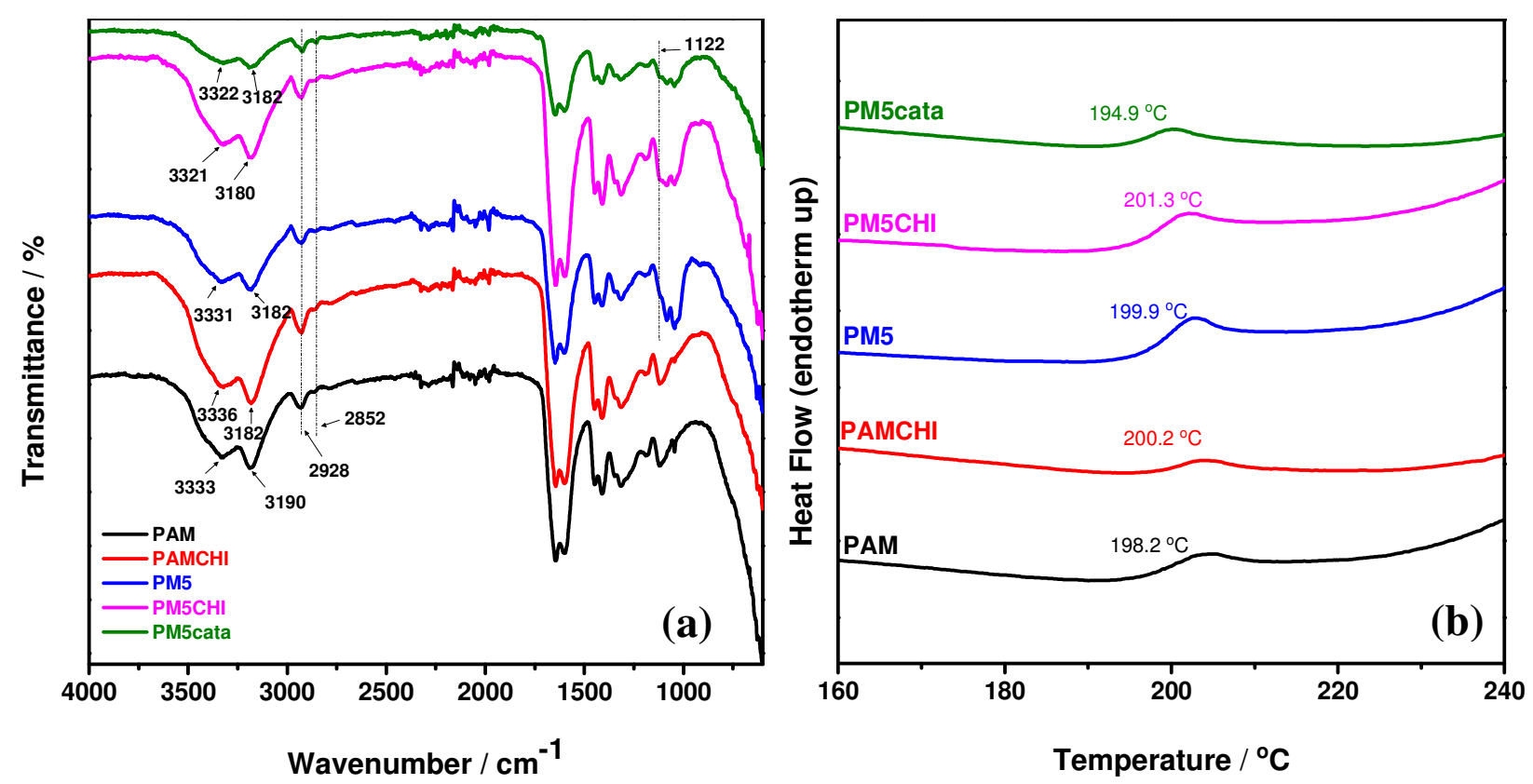

Figure 4. (a) FTIR spectra and (b) DSC traces of PM5 and PM5CHI dry samples as well as their control samples, PAM, PAMCHI and PM5cata.

To confirm the above postulation, FTIR and DSC were performed to investigate the chemical structure and the intermolecular interactions of the two nanocomposite hydrogels in comparison with their control samples (Figure 4). There were peaks at $2852 \mathrm{~cm}^{-1}$ and $2928 \mathrm{~cm}^{-1}$. The former was characteristic of tertiary $\mathrm{CH}$ and secondary $\mathrm{CH}_{2}$, while the latter was characteristic of primary $\mathrm{CH}_{3}$ for all samples. ${ }^{46,47}$ By comparing the relative intensity of $\mathrm{CH}$ and $\mathrm{CH}_{2}$ to $\mathrm{CH}_{3}$, it was found that the relative intensity values for PM5 and PM5CHI were lower than that of PM5cata. Considering that $\mathrm{CH}_{2}$ was stable during polymerization, ${ }^{43,48}$ this result implied that the amount of tertiary $\mathrm{CH}$ bonds reduced, confirming the chain transfer reaction and chain branching proposed by others for branched PAM. ${ }^{49}$ During PAM polymerization, chain transfer reaction is rare when the polymerization temperature is below $50{ }^{\circ} \mathrm{C}$, but cannot be ignored at a temperature above 50 ${ }^{\circ} \mathrm{C}$ which leads to a branched PAM. ${ }^{43,48}$ As shown in Scheme 3 (a), when the hydrogen atoms from 
the tertiary $\mathrm{CH}$ are eliminated under the attack of free radicals, the polymer radicals will be formed, onto which chain propagation will lead to branches.

For the peaks characteristic for amine N-H stretching at around $3190 \mathrm{~cm}^{-1}$ and $3333 \mathrm{~cm}^{-1}$ for PAM, they all shifted to a low wavenumber for PM5, PM5CHI and PM5cata compared to those of PAM and PAMCHI, confirming the hydrogen bonding between PAM and MMT. ${ }^{22}$ The peaks at $1122 \mathrm{~cm}^{-1}$ was characteristic for C-O-C stretching. By comparing the relative intensity of C-O$\mathrm{C}$ to $\mathrm{N}-\mathrm{H}$, it was observed that the value for PM5CHI was larger than those of PM5 and PM5cata, indicating the chemical grafting of PAM onto the chitosan backbone through etherification depicted in Scheme 3 (b). ${ }^{19,50}$

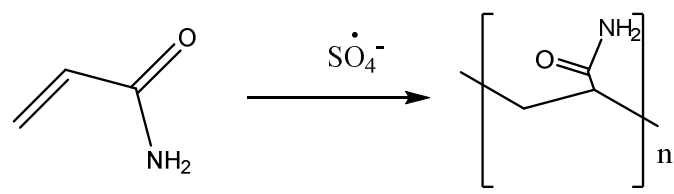

(a)

Free radical polymerization of AM

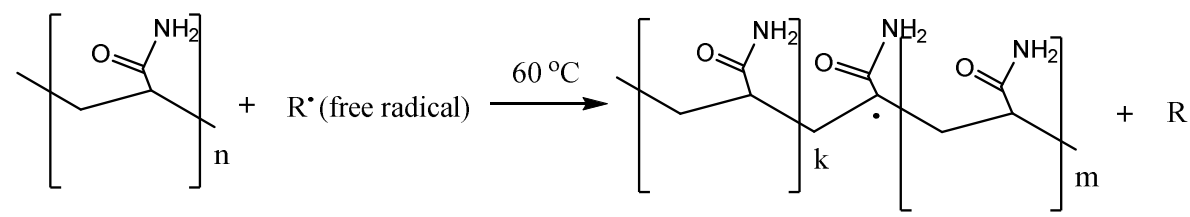

Chain transfer reaction to polymer backbone

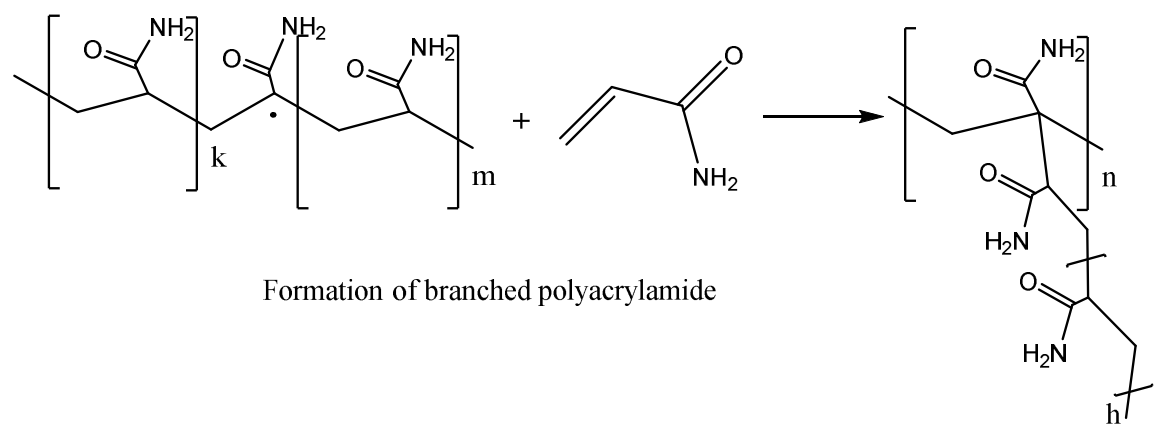




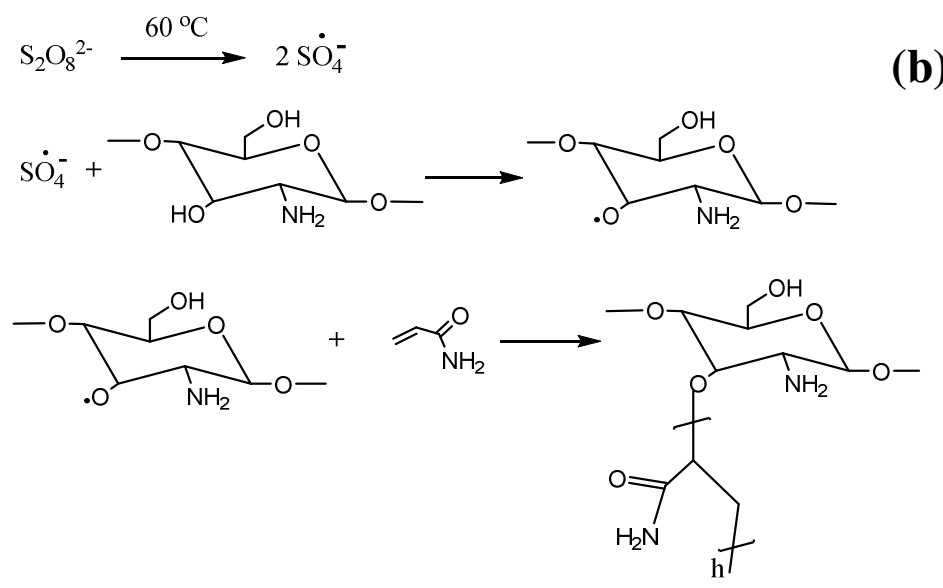

Scheme 3. Mechanisms for (a) Free Radical Polymerization, Chain Transfer and the Formation of Branched Polyacrylamide, and (b) Radical Graft Polymerization ${ }^{50}$ of AM onto Chitosan.

The presence of branched PAM, PAM-grafted-chitosan and the interfacial interactions within the nanocomposite hydrogels was confirmed by DSC results (Figure 4 (b)). The glass transition temperature $\left(\mathrm{T}_{\mathrm{g}}\right)$ of the branched PAM synthesized at $60{ }^{\circ} \mathrm{C}$ was $198.2^{\circ} \mathrm{C}$, which was higher than the $\mathrm{T}_{\mathrm{g}}$ of the single-chain polyacrylamide nanoglobules $\left(\sim 191{ }^{\circ} \mathrm{C}\right)$ and the bulk polyacrylamide $\left(188^{\circ} \mathrm{C}\right)$, scanned at a rate of $20^{\circ} \mathrm{C} .{ }^{51}$ These different $\mathrm{T}_{\mathrm{g}}$ values were attributable to the difference in chemical structure, chain entanglement degree ${ }^{51}$ and experimental conditions. PAMCHI showed a higher $\mathrm{T}_{\mathrm{g}}\left(200.2^{\circ} \mathrm{C}\right)$ due to the participation of chitosan in the reaction. ${ }^{19}$ The presence of MMT in PM5 increased the $\mathrm{T}_{\mathrm{g}}$ of PAM to $199.9^{\circ} \mathrm{C}$. Similarly, the $\mathrm{T}_{\mathrm{g}}$ of PM5CHI $\left(201.3{ }^{\circ} \mathrm{C}\right)$ was slightly higher than that of PAMCHI. Both results confirmed strong PAMM-MMT hydrogen bonding in the nanocomposite hydrogels. ${ }^{22}$ The higher $\mathrm{T}_{\mathrm{g}}$ displayed by PM5CHI, compared to that of PM5, also gave evidence of stronger interfacial interactions in the former. In addition, the $\mathrm{T}_{\mathrm{g}}$ of PM5cata with linear PAM chains was $194.9^{\circ} \mathrm{C}$, lower than the values for both PM5 and PM5CHI which contained branched PAM chains. Considering the similarity in the chemical composition of these 
three nanocomposites, the higher $\mathrm{T}_{\mathrm{g}}$ values of PM5 and PM5CHI could be attributed to the denser crosslinks arising from the branched chains and/or PAM-grafted chitosan and hence reduced chain mobility. ${ }^{43,52}$

As discussed earlier, PM5CHI displayed higher tensile strength, strain at break and tensile energy at break than PM5. The chemical grafting of some PAM macromolecular chains onto chitosan backbone, together with the stronger electrostatic forces between chitosan and MMT compared to the hydrogen bonding between PAM and MMT, accounted for the superior mechanical properties in PM5CHI.

Similar results could also be achieved by using another polysaccharide such as starch or $k$ carrageenan instead of chitosan to prepare nanocomposite hydrogels (Figure S1, Table S1 and Table S2), in which chain branching and chemical grafting of PAM onto the hydroxyl groups of the two polysaccharides also occurred due to the high reaction temperature. By comparing the relative intensity of $\mathrm{CH}$ and $\mathrm{CH}_{2}\left(2852 \mathrm{~cm}^{-1}\right)$ to $\mathrm{CH}_{3}\left(2928 \mathrm{~cm}^{-1}\right)$, it was found that the values for PAM-starch treated MMT (PM5S) and PAM- $k$-carrageenan treated MMT (PM5K) nanocomposite hydrogels were lower than that of PM5cata. The tensile strengths for PM5S and PM5K were 89.2 and $92.0 \mathrm{kPa}$, respectively, which were significantly higher than that of PM5.

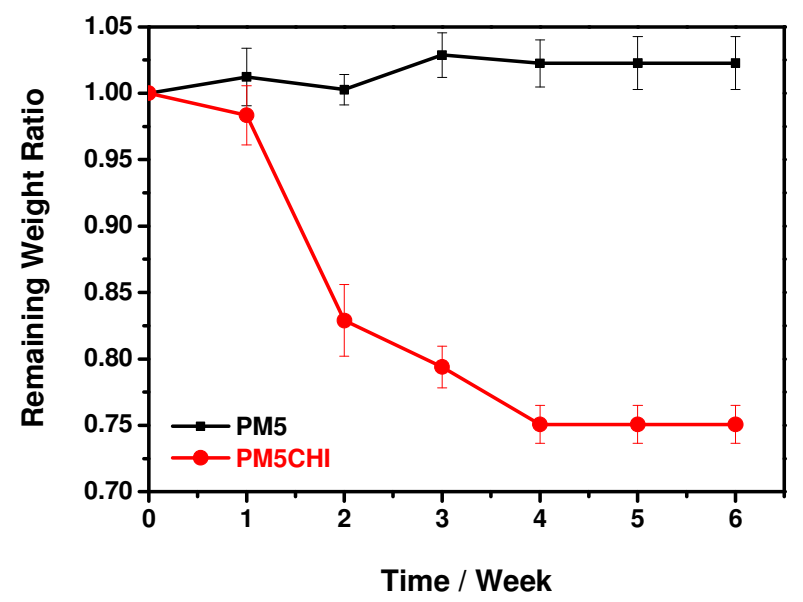

Figure 5. In vitro degradation results of PM5 and PM5CHI hydrogels. 
As stated above, the graft polymerization of AM onto chitosan backbone was important to the mechanical properties of PM5CHI. This could be further confirmed by the in vitro degradation results as shown in Figure 5. The weight of PM5 hardly changed because both PAM and MMT platelets were stable in lysozyme-containing PBS. The slight weight gain by PM5 after two weeks was probably because the hydrogel samples absorbed ions from PBS solution. In contrast, PM5CHI decreased its weight by $25 \%$ in 5 weeks, which was attributable to the presence of chitosan in the hydrogel. Lysozyme is efficient to degrade polysaccharides including chitosan by hydrolyzing the glycosidic linkages via its hexameric sugar ring at binding sites. ${ }^{37}$ Remarkably, even with such a low weight percentage of chitosan in respect to PAM, 0.88\%, PM5CHI degraded up to $25 \%$ of its original weight. This proved that chitosan not only served as the surface modifier of MMT platelets but also the backbone onto which PAM polymer chains chemically grafted. These degradation results implied that a small amount of chitosan could lead to substantial biodegradation, and so biodegradability of PAM hydrogels could be potentially tailored by varying the amount of chitosan.

Cyclic tensile tests were also carried out for PM5 and PM5CHI to investigate the resilience and hysteresis behaviour of the hydrogels. Their representative testing curves are shown in Figure 6 . It could be seen that most of the curves were not smooth; the zigzag was due to the intrinsic properties of the hydrogels rather than the machine noise (Figure S2). Intermolecular friction and disentanglement of PAM chains ${ }^{53-54}$ were likely to be the main reasons for the zigzag in the curves of Figure 6. Under stretching, PAM chains slid across each other and MMT platelets, during which PAM-PAM and PAM-MMT hydrogen bonds broke and reformed repeatedly, causing fluctuation of the tensile curves..$^{22,54}$ 

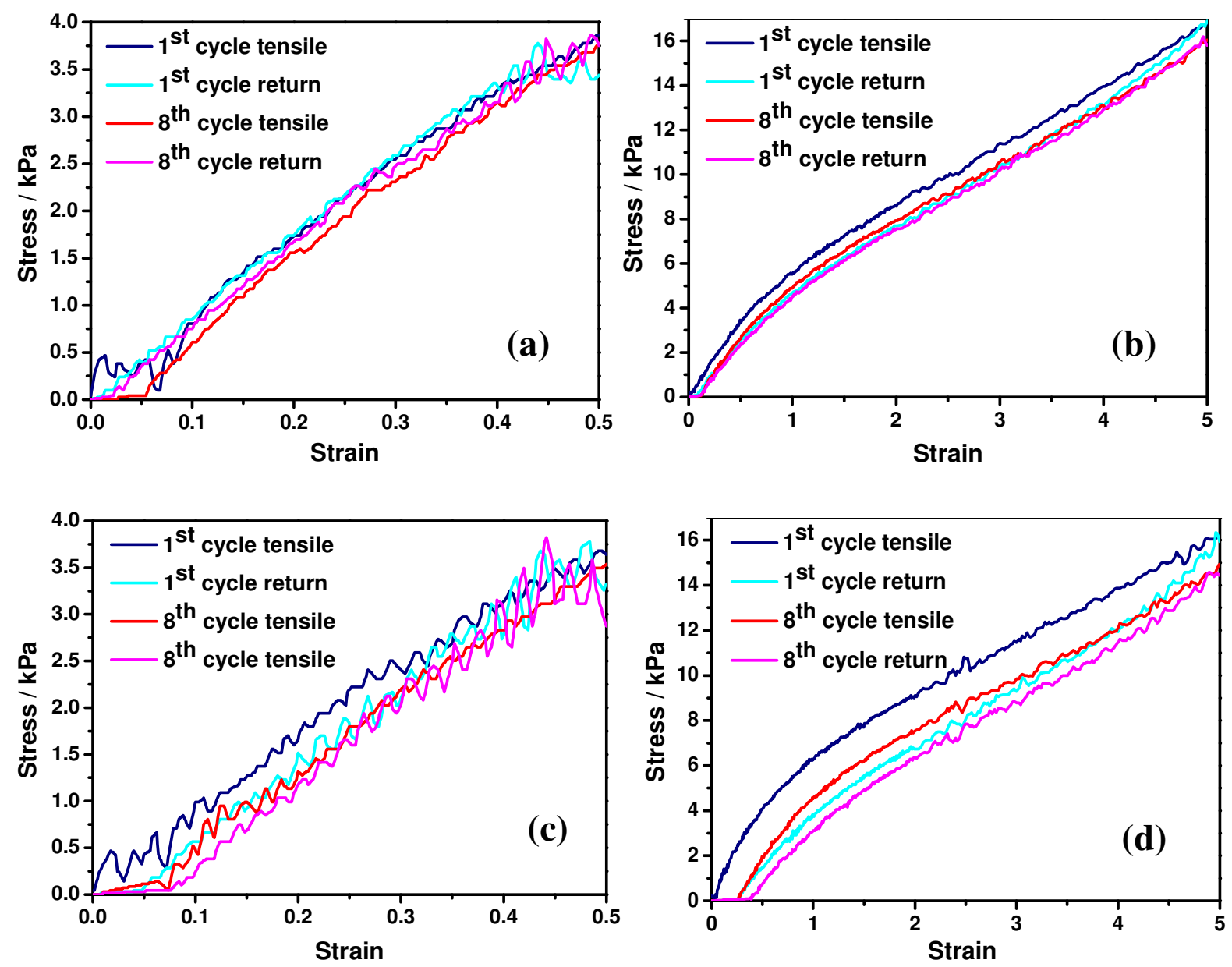

Figure 6. Representative cyclic tensile testing curves of the hydrogels. Cyclic tension to (a) $50 \%$ of PM5, (b) 500\% of PM5, (c) 50\% of PM5CHI and (d) 500\% of PM5CHI.

Table 2. Hysteresis ratios of PM5 and PM5CHI hydrogels.

\begin{tabular}{l|l|l|l}
\hline & & PM5 & \multicolumn{2}{|c}{ PM5CHI } \\
\hline Strain & Cycle & \multicolumn{2}{|c}{ Hysteresis Ratio, $h$} \\
\hline 0.5 & $1^{\text {st }}$ Cycle & $-0.007 \pm-0.002$ & $0.10 \pm 0.01$ \\
\hline 0.5 & $8^{\text {th }}$ Cycle & $-0.05 \pm-0.01$ & $0.04 \pm 0.01$ \\
\hline 5 & $1^{\text {st }}$ Cycle & $0.09 \pm 0.01$ & $0.20 \pm 0.01$ \\
\hline 5 & $8^{\text {th }}$ Cycle & $0.04 \pm 0.01$ & $0.10 \pm 0.01$ \\
\hline
\end{tabular}


As we can see from Figure $6(a, b)$ and Table 2, in terms of PM5, the stretching and return curves for the first cycle to $50 \%$ strain overlapped each other nicely with no hysteresis, which suggested that under a maximum strain of 50\%, PM5 was very resilient and could recover instantly on the return. After 8 cycles, the material could still recover quickly meaning that even undergoing 8 cycles, the energy dissipation by the friction of PAM chains ${ }^{22,44}$ was still negligible, showing nearly no hysteresis.

As for a cyclic tensile strain up to $500 \%$ which was beyond the yield point, hysteresis was still quite low, being $8 \%$ for the first cycle. Based on the chain length theory ${ }^{55}$ the multiple and relatively strong PAM-MMT hydrogen bonds remained to preserve the resilience even after a strain of $500 \%$. This value was, however, slightly higher when compared to cyclic tension to $50 \%$, probably because many PAM-MMT hydrogen bonds broke during stretching and the disassociated PAM-MMT hydrogen bonds could not recover fully instantly. With increasing cycle number, MMT platelets tended to orient parallel to the tensile direction so that hysteresis became lower. According to a previous study, ${ }^{44}$ under a large deformation, polymer chains were highly tensioned and clay platelets tended to orient horizontally in respect to the stretching direction. On the return cycle, MMT platelets rotated and overcame the steric effect and friction force associated with the adjacent PAM chains for recovery. ${ }^{25}$

Hysteresis was also investigated for PM5CHI. In Figure 6 (c,d) and Table 2, for cyclic tension to $50 \%$, the $1^{\text {st }}$-cycle hysteresis was still quite low, indicating fairly good resilience. Similar to PM5, the low hysteresis of PM5CHI could be due to the covalent bonds brought by PAM-graftedchitosan, as well as the strong multiple hydrogen bonds between branched PAM and MMT. However, unlike PM5, the $1^{\text {st }}$-cycle hysteresis of PM5CHI by cyclic tension to 50\% could not be ignored, which could be attributed to the electrostatic interactions between the negatively charged 
MMT and the surface-adsorbed positively charged chitosan chains. During cyclic tension to a strain of 50\%, chitosan chains were stretched and aligned across the MMT surface which showed a hysteresis of $10 \%$ probably because of energy dissipation owing to the alignment of chitosan chains and the friction occurred at the chitosan-MMT interface. ${ }^{32,56}$ When unloaded, the detached chitosan-MMT electrostatic interactions were not able to recover quickly. ${ }^{32,56}$ After each cycle, chitosan-MMT electrostatic interactions could only partially recover within seconds during the return cycle. Subsequently, as more tensile cycles were experienced, fewer chitosan-MMT electrostatic interactions broke and contributed to hysteresis. This, together with orientation of MMT and stretching of polymer molecules as discussed for PM5, accounted for the lower hysteresis ratio found for the $8^{\text {th }}$ cycle when compared to the value for the $1^{\text {st }}$ cycle. When the cyclic maximum strain increased from $50 \%$ to $500 \%$, significant unzipping of chitosan-MMT electrostatic interactions increased hysteresis. Under high strains, more chitosan chains detached from the MMT platelets, resulting in more energy dissipation and thus higher hysteresis. Like cyclic tension to $50 \%$, the hysteresis also became lower after 8 cycles.

The hysteresis ratios of the PM5 reported herein are much lower than most hydrogels described in the literature. $22,57,58$ They are also lower than or comparable to the lowest hysteresis ratios reported to date for PAM-clay nanocomposite hydrogels, namely PAM-synthetic hectorite hydrogels, ${ }^{25}$ which showed a hysteresis ratio of 0.19 for the $1^{\text {st }}$ cycle and 0.09 for the $2^{\text {nd }}$ cycle to a maximum strain of $1000 \%$. As stated above, the low hysteresis could be attributed to strong polymer-clay bonding, ${ }^{11,25,59}$ and ample covalent bonds due to polymer branching from the high temperature reaction. These strong and multiple bonds acted as memory sites for fast shape recovery. 
By comparing hysteresis of PM5CHI to that of other double-network PAM hydrogels without clay, ${ }^{57,60}$ PM5CHI still showed significantly lower hysteresis of 0.20 for the $1^{\text {st }}$ cycle to $500 \%$, while 0.60 or even higher hysteresis was obtained in those two studies. This was due to branched PAM, PAM-grafted-chitosan and the strong and multiple PAM-MMT hydrogen bonding as discussed before. Similar to PM5, PM5CHI was characterized with ample memory sites, leading to good resilience. Both PM5CHI and PM5 were able to recover their original shape fully in 1 minute (Figure S3), which was much faster than the work reported by others, where 120 minutes was necessary for recovery of randomly copolymerized polyampholytes double-network hydrogels, ${ }^{60}$ and 1 day at $80{ }^{\circ} \mathrm{C}$ was required for recovery to $74 \%$ of the original shape of PAMalginate double network hydrogels. ${ }^{57}$

The crosslinking density was estimated by the classic kinetic theory of rubber elasticity to depict the elasticity of polymer nanocomposite hydrogels ${ }^{61-62}$. The formula was given below.

$$
\mathrm{F}=\mathrm{N}^{*} \mathrm{RT}\left(\alpha-\alpha^{-2}\right)
$$

where $\mathrm{F}, \mathrm{N}^{*}, \mathrm{R}, \mathrm{T}$ and $\alpha$ are the force per unit original cross-sectional area of the hydrogels, effective crosslinking density, gas constant, absolute temperature and the extension ratio, respectively. Here $\alpha$ was set to be 2 , corresponding to a strain of $100 \%$ and $\mathrm{T}=298.15 \mathrm{~K}$. From Figure 2, $\mathrm{N}^{*}$ was calculated as $1.27 \mathrm{~mol} \mathrm{~m}^{-3}$ for the as-prepared PM5 samples and $1.43 \mathrm{~mol} \mathrm{~m}^{-3}$ for the as-prepared PM5CHI samples.

Comparing PM5 with PM5CHI, the latter showed a higher crosslinking density, in line with the mechanical properties discussed earlier, and it could be attributed to the number of reactive sites. In the case of PM5, PAM interacted with MMT platelets; while in the case of PM5CHI, PAM interacted with both MMT platelets and chitosan. Apart from PAM-MMT hydrogen bonding which was also presented in PM5, chitosan offered additional crosslinking sites for PM5CHI and thus higher crosslinking density. 

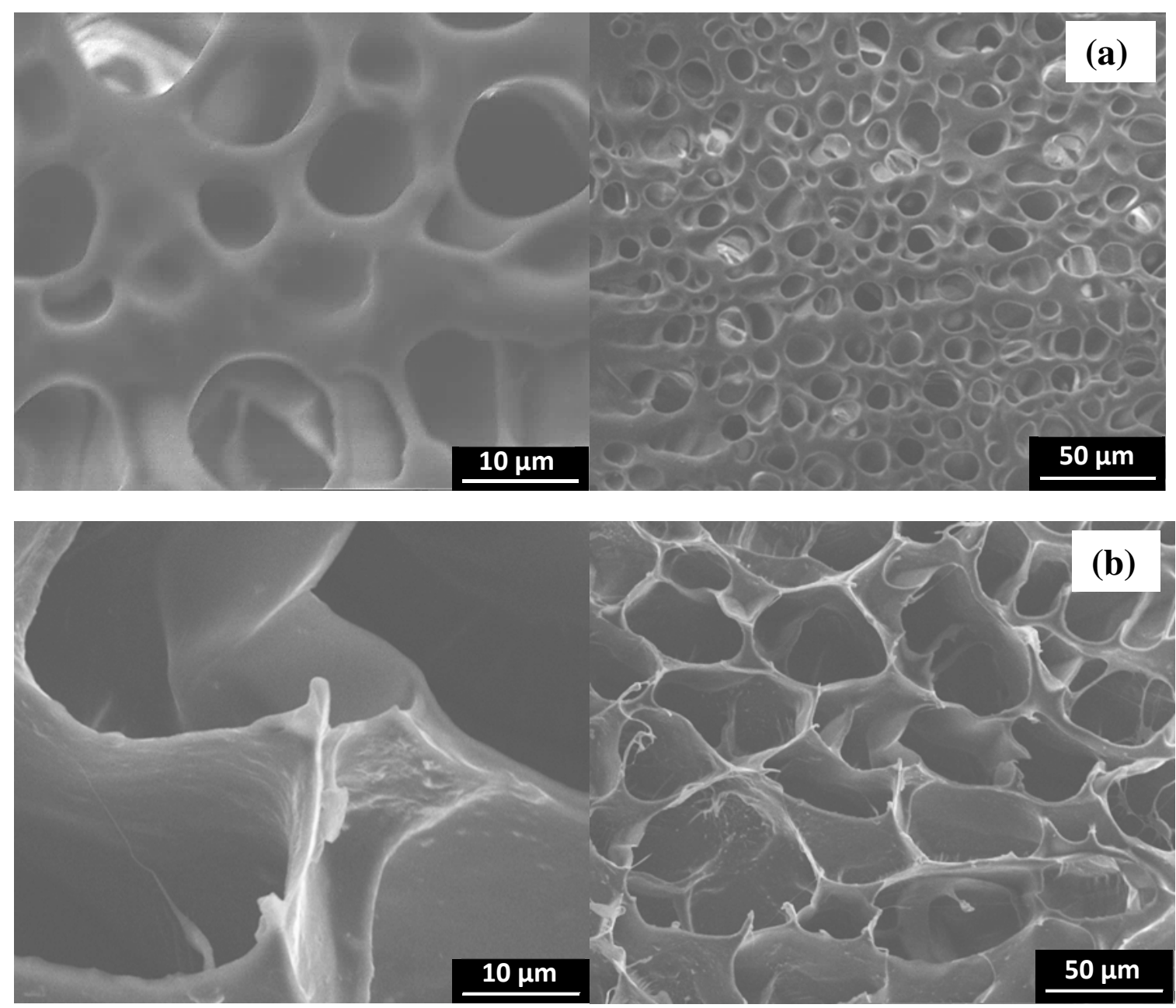

Figure 7. SEM images of the cross-sectional surfaces of freeze-dried (a) PM5 and (b) PM5CHI.

To understand the internal morphology of the nanocomposite hydrogels, the cross-sectional surface of freeze-dried PM5 and PM5CHI samples was observed by SEM. As shown in Figure 7, both hydrogels showed interconnected porous structure. The pore size of PM5CHI $(51.2 \pm 7.2 \mu \mathrm{m})$ was larger than that of PM5 $(7.7 \pm 2.1 \mu \mathrm{m})$, which could be attributed to the chitosan chains on part of the MMT surface. Cationic chitosan chains tended to repulse each other, forming interconnected porous structure with large pore sizes. ${ }^{63}$ 


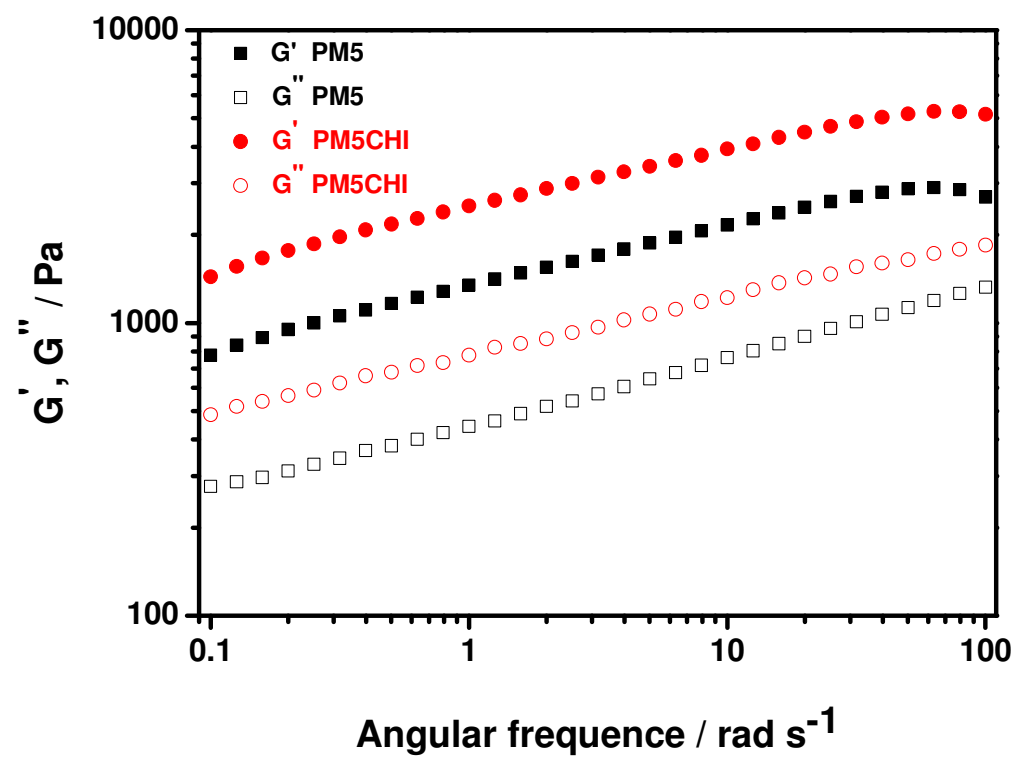

Figure 8. Storage moduli G' and loss moduli G" of PM5 and PM5CHI hydrogels.

Rheological properties were depicted in Figure 8 for PM5 and PM5CHI. The storage modulus of both hydrogels increased slightly with increasing angular frequency. Besides, their storage moduli were significantly larger than loss moduli, confirming that both hydrogels were mainly elastic instead of viscous polymer solution. ${ }^{64}$ Comparing the storage moduli of PM5 and PM5CHI, the incorporation of chitosan nearly doubled the storage moduli. Chitosan was responsible for introducing chitosan-MMT electrostatic interactions and chitosan-PAM chemical grafting, which were much stronger than the PAM-MMT hydrogen bonding as discussed previously.

Mechanical properties of fully swollen nanocomposite hydrogels. Prior to the mechanical testing of the fully swollen nanocomposite hydrogels, swelling tests on the freezedried hydrogels were performed in distilled water at room temperature. As can be seen from Figure 
9 (a) and Table 3, both PM5 and PM5CHI were able to absorb a large amount of water with a swelling ratio over 20 after several days. The slightly decreased swelling ratio after 100 hours for PM5 was due to the dissolution of some small molecular weight substances into water. The incorporation of chitosan significantly reduced the equilibrium swelling ratio. This was because the higher crosslinking density of PM5CHI restricted the swelling of PAM chains to a greater extent when compared to PM5. The swelling ratios of both PM5 and PM5CHI were lower than that of the PAM-MMT hydrogel containing the same clay content reported in the literature. ${ }^{22}$ This may be mainly attributed to the high branching of the polymer in the former two hydrogels, which caused shorter and less swellable PAM chains compared to the linear PAM chains in the latter.
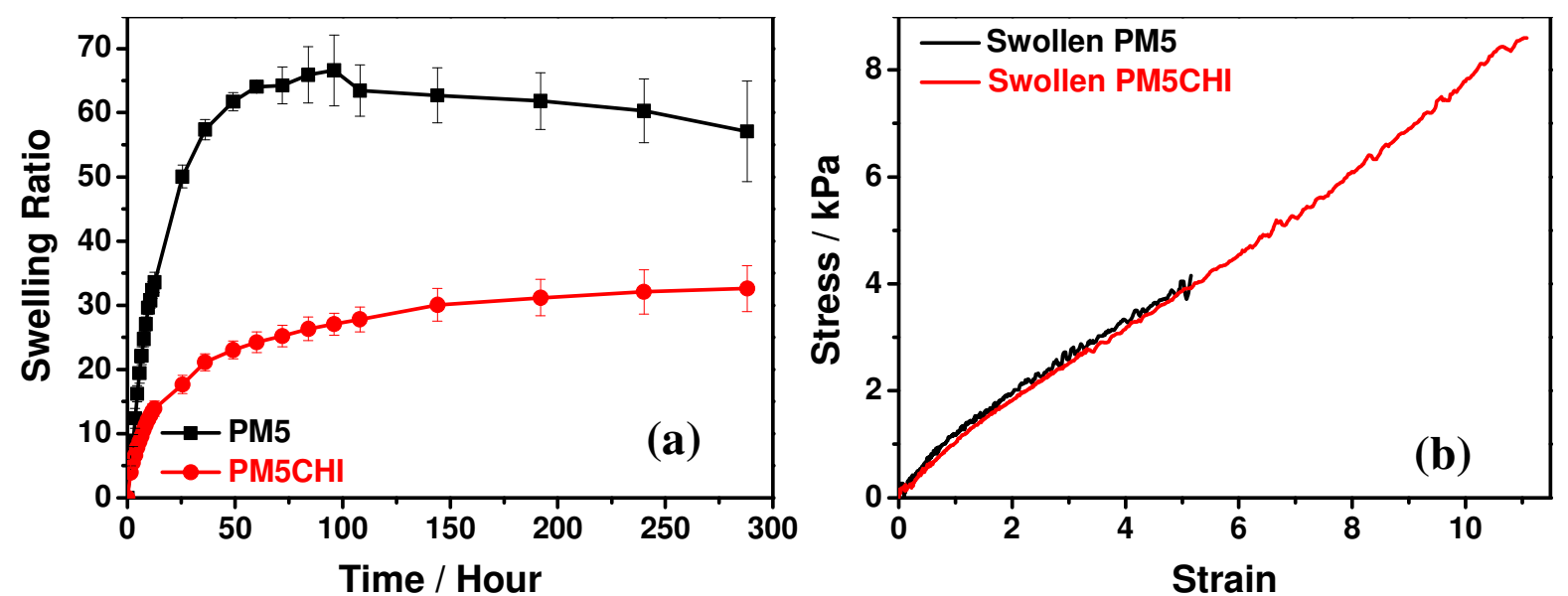

Figure 9. (a) Swelling ratio as a function of immersion time in distilled water at $25{ }^{\circ} \mathrm{C}$ for PM5 and PM5CHI hydrogels, and (b) tensile curves of PM5 and PM5CHI hydrogels swollen in PBS at $37^{\circ} \mathrm{C}$ for 2 weeks. 
Table 3. Tensile properties of the hydrogels fully swollen in PBS at $37{ }^{\circ} \mathrm{C}$ for 2 weeks.

\begin{tabular}{llllll}
\hline Samples & $\begin{array}{l}\text { Swelling } \\
\text { ratio, } \varphi\end{array}$ & $\begin{array}{l}\text { Young's } \\
\text { modulus } / \mathrm{kPa}\end{array}$ & $\begin{array}{l}\text { Tensile } \\
\text { strength } / \mathrm{kPa}\end{array}$ & $\begin{array}{l}\text { Strain at } \\
\text { break }\end{array}$ & $\begin{array}{l}\text { Energy at break } \\
/ \mathrm{kJ} \mathrm{m}^{-3}\end{array}$ \\
\hline PM5 & $64 \pm 2$ & $1.2 \pm 0.2$ & $4.5 \pm 0.6$ & $5.3 \pm 0.2$ & $13.1 \pm 2.0$ \\
\hline PM5CHI & $32 \pm 3$ & $1.2 \pm 0.3$ & $8.6 \pm 0.8$ & $11.1 \pm 0.3$ & $48.0 \pm 3.0$ \\
\hline
\end{tabular}

For mechanical tests on the swollen samples, the as-prepared hydrogels were submerged in a PBS solution at $37{ }^{\circ} \mathrm{C}$ for two weeks, allowing for the full swelling, to study their mechanical properties in a simulative body environment. The results were shown in Figure 9 (b) and Table 3. The fully swollen hydrogels displayed significantly inferior mechanical properties compared to their as-prepared hydrogels because of the much higher water contents. According to swelling tests, swollen PM5 contained 99.7 wt\% of water and swollen PM5CHI contained 99.4 wt\% of water (while these values may vary in the simulative body environment). Further, the entangled polymer chains were more extended after swelling and so lost part of their flexibility. However, compared to the conventional chemically crosslinked PAM hydrogels, which usually become very brittle and fragile in the fully swollen state, PM5 and PM5CHI could still be stretched to a strain over $500 \%$. There are two reasons: (1) the relatively uniform crosslinking gave rise to the high extensibility of hydrogels, and (2) PAM branching and/or the grafting of PAM onto chitosan chains contributed to the strength of the fully swollen hydrogels. The swollen PM5CHI displayed a much higher tensile strength and strain at break than those of PM5, and it could also withstand a compression load of $1 \mathrm{kN}$ without failure (Figure S4). The superior mechanical properties of the fully swollen PM5CHI over PM5 may be attributed to two factors. One is the much lower water content as shown by the swelling test, while the other is the strong bonding within the hydrogel due to the presence of chitosan as mentioned above. 
These results indicated that it was possible to achieve high strength, high extensibility and high resilience with PAM nanocomposite hydrogels consisting of positively charged chitosan chains and negatively charged clay nanoparticles. This work may be extended to other nanoparticles with negative charges or neutral nanoparticles, such as nano-hydroxyapatite, ${ }^{65}$ functionalized with an anionic chemical.

Fabrication versatility of nanocomposite hydrogels. The nanocomposite hydrogels could be fabricated into different shapes and dimensions, e.g. rods and balls, by using different moulds, as shown in Figure 10 (a). They could also be spun into microfibers using pressurized gyration. Figure 10 (b) shows proof-of-concept results, using the precursor route, from the pressurized gyration process at $36000 \mathrm{rpm}$ rotating speed and $0.1 \mathrm{MPa}$ working pressure. The fiber diameter measured at this condition was $5.5 \pm 0.6 \mu \mathrm{m}$. Varying the spinning conditions, e.g. the pressure, may lead to different fiber morphologies such as different fiber diameters, fiber strands and/or twisted fibers. ${ }^{32,62,66}$ These fibers could be further polymerized to obtain nanocomposite hydrogel fibers.

The versatility of fabricating the nanocomposite hydrogels into different forms, shapes and dimensions will enable the hydrogels to be applied in a variety of applications such as artificial tissues, ${ }^{7,67-68}$ stimuli-responsive and superabsorbent functional nanocomposites, ${ }^{19,22,50}$ drug delivery, ${ }^{8}$ and actuators. ${ }^{69}$ In particular, hydrogel fibers may be beneficial in tissue reconstruction, wound dressing, smart textiles and heavy metal uptake applications due to their high specific surface area. ${ }^{14}$ 


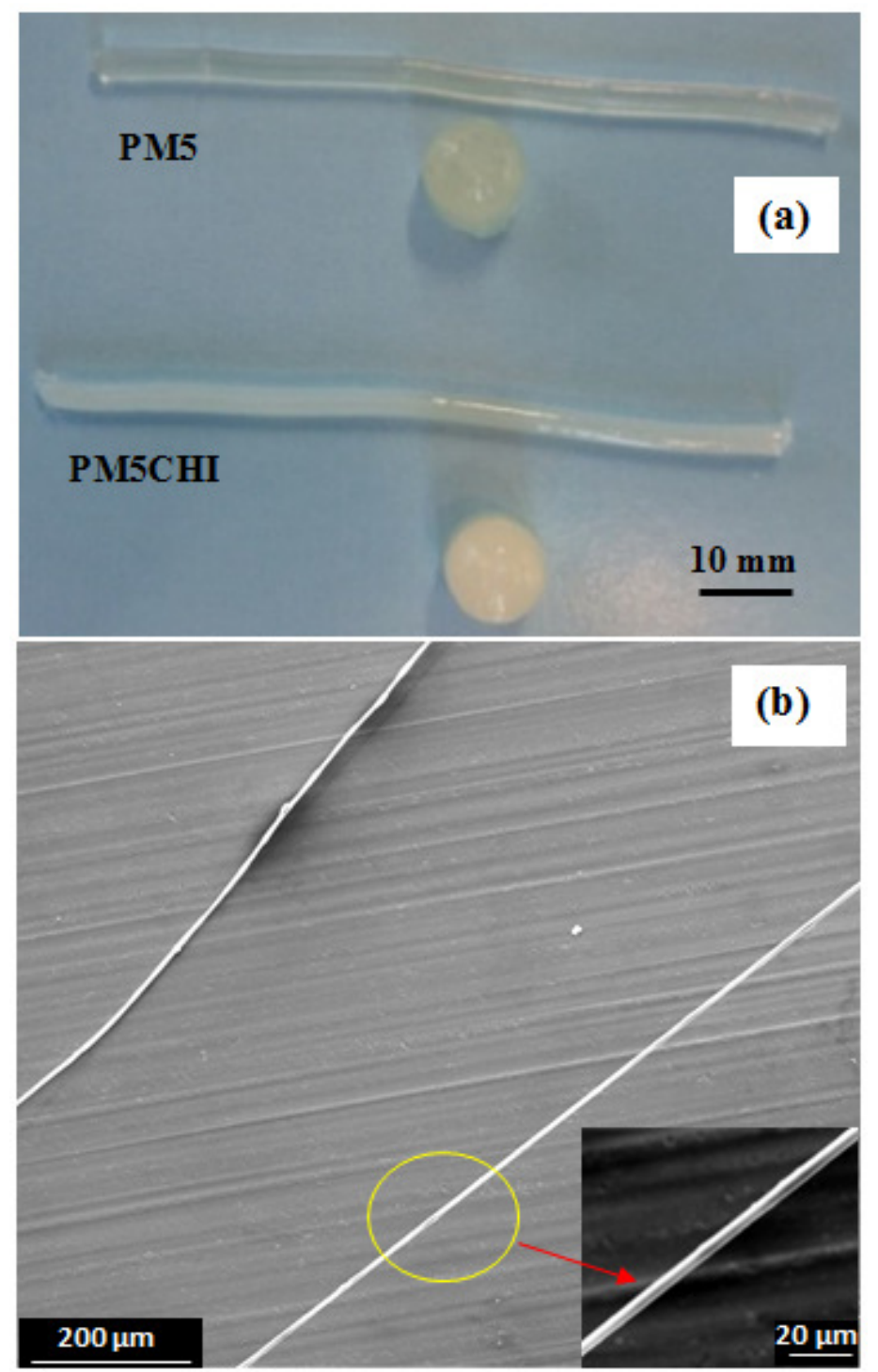

Figure 10. (a) Photographs of PM5 and PM5CHI hydrogels in different shapes (rods and balls),

(b) SEM images of PM5CHI microfibers obtained at $36000 \mathrm{rpm}$ rotating speed and $0.1 \mathrm{MPa}$ working pressure. The inset in (b) shows the corresponding higher magnification image.

\section{CONCLUSIONS}


Highly stretchable PAM nanocomposite hydrogels with low hysteresis were prepared with the presence of a small amount of MMT or chitosan-MMT at an elevated temperature. Both PM5 and PM5CHI displayed relatively high tensile strength, strain at break, fast shaper recovery and low hysteresis. Comparing PM5 with PM5CHI, the latter showed higher tensile strength and elongation at break but slightly larger hysteresis. The tensile properties deteriorated after the hydrogels had been fully swollen in PBS for 2 days at $37{ }^{\circ} \mathrm{C}$. Yet, the fully swollen PM5CHI could still be stretched by over 10 times and able to withstand a compressive load of $1 \mathrm{kN}$. PM5CHI had a lower equilibrium water swelling ratio than PM5. A $25 \%$ of weight loss was achieved by PM5CHI by biodegradation while no weight loss was observed by PM5. The excellent mechanical properties of the hydrogels were attributed to the strong interactions within the hydrogels brought by chain branching, multiple hydrogen bonding, covalent bonding and/or electrostatic force, as confirmed by XRD, FTIR, DSC, in vitro degradation test, SEM and the replacement of chitosan with another polysaccharide, namely starch or $k$-carrageenan, in the synthesis. The rheological results confirmed elastic nature of the hydrogels. The hydrogels may be fabricated into various forms and shapes, including microfibers using pressurized gyration via the precursor route. The nanocomposite hydrogels reported herein illustrated an effective way to developing advanced polymer nanocomposite hydrogels with superior mechanical properties and versatile fabrication capability, which may find potential applications in artificial tissues, functional films, drug delivery systems, actuators and beyond.

\section{ASSOCIATED CONTENT}

Supporting Information. Tensile curves and table of tensile properties of PM5S and PM5K hydrogels; cyclic tensile curves and table of cyclic tensile properties of PM5S and PM5K 
hydrogels; tensile curve of a wet polyvinyl alcohol sample; loading-unloading curves of PM5 and PM5CHI hydrogels at a strain of 300\%; compressive curves and photographs of fully swollen PM5 and PM5CHI samples before, during and after compression tests.

\section{REFERENCES}

(1) Kabiri, K.; Omidian, H.; Zohuriaan-Mehr, M. J.; Doroudiani, S., Superabsorbent Hydrogel Composites and Nanocomposites: A Review. Polym. Composite. 2011, 32, $277-289$.

(2) Huang, T.; Xu, H. G.; Jiao, K. X.; Zhu, L. P.; Brown, H. R.; Wang, H. L., A Novel Hydrogel with High Mechanical Strength: A Macromolecular Microsphere Composite Hydrogel. Adv. Mater. 2007, 19, 1622-1626.

(3) Huang, Y.; Dong, R.; Zhu, X.; Yan, D., Photo-Responsive Polymeric Micelles. Soft Matter 2014, 10, 6121-6138.

(4) Huang, Y.; Qiu, F.; Shen, L.; Chen, D.; Su, Y.; Yang, C.; Li, B.; Yan, D.; Zhu, X., Combining Two-Photon-Activated Fluorescence Resonance Energy Transfer and near-Infrared Photothermal Effect of Unimolecular Micelles for Enhanced Photodynamic Therapy. ACS Nano 2016, 10, 10489-10499.

(5) Saxena, A. K., Synthetic Biodegradable Hydrogel (Pleuraseal) Sealant for Sealing of Lung Tissue after Thoracoscopic Resection. J. Thorac. Cardiovasc. Surg. 2010, 139, 496-497.

(6) Chen, X.; Martin, B. D.; Neubauer, T. K.; Linhardt, R. J.; Dordick, J. S.; Rethwisch, D. G., Enzymatic and Chemoenzymatic Approaches to Synthesis of Sugar Based Polymer and Hydrogels. Carbohydr. Polym. 1995, 28, 15-21.

(7) Frydrych, M.; Roman, S.; Green, N. H.; MacNeil, S.; Chen, B. Q., Thermoresponsive, Stretchable, Biodegradable and Biocompatible Poly(glycerol sebacate)-Based Polyurethane Hydrogels. Polym. Chem. 2015, 6, 7974-7987.

(8) Hoare, T. R.; Kohane, D. S., Hydrogels in Drug Delivery: Progress and Challenges. Polymer 2008, 49, 1993-2007.

(9) Sirousazar, M.; Kokabi, M.; Hassan, Z. M.; Bahramian, A. R., Polyvinyl Alcohol/NaMontmorillonite Nanocomposite Hydrogels Prepared by Freezing-Thawing Method: Structural, Mechanical, Thermal, and Swelling Properties. J. Macromol. Sci. Phys. 2012, 51, 1335-1350.

(10) Li, L. L.; Wang, Y. Q.; Pan, L. J.; Shi, Y.; Cheng, W.; Shi, Y.; Yu, G. H., A Nanostructured Conductive Hydrogels-Based Biosensor Platform for Human Metabolite Detection. Nano Lett. 2015, 15, 1146-1151.

(11) Haraguchi, K.; Takehisa, T., Nanocomposite Hydrogels: A Unique Organic-Inorganic Network Structure with Extraordinary Mechanical, Optical, and Swelling/De-Swelling Properties. Adv. Mater. 2002, 14, 1120-1124.

(12) Haraguchi, K.; Takehisa, T.; Fan, S., Effects of Clay Content on the Properties of Nanocomposite Hydrogels Composed of Poly(N-isopropylacrylamide) and Clay. Macromolecules 2002, 35, 10162-10171.

(13) Haraguchi, K.; Li, H. J.; Matsuda, K.; Takehisa, T.; Elliott, E., Mechanism of Forming Organic/Inorganic Network Structures During in-Situ Free-Radical Polymerization in PNIPAMClay Nanocomposite Hydrogels. Macromolecules 2005, 38, 3482-3490. 
(14) Ahmed, E. M., Hydrogel: Preparation, Characterization, and Applications: A Review. J. Adv. Res. 2015, 6, 105-121.

(15) Smith, E. A.; Oehme, F. W., Acrylamide and Polyacrylamide: A Review of Production, Use, Environmental Fate and Neurotoxicity. Rev. Environ. Health 1991, 9, 215-28.

(16) Alexandre, M.; Dubois, P., Polymer-Layered Silicate Nanocomposites: Preparation, Properties and Uses of a New Class of Materials. Mat. Sci. Eng. R. 2000, 28, 1-63.

(17) Guilherme, M. R.; Fajardo, A. R.; Moia, T. A.; Kunita, M. H.; Goncalves, M. D.; Rubira, A. F.; Tambourgi, E. B., Porous Nanocomposite Hydrogel of Vinyled MontmorilloniteCrosslinked Maltodextrin-Co-Dimethylacrylamide as a Highly Stable Polymer Carrier for Controlled Release Systems. Eur. Polym. J. 2010, 46, 1465-1474.

(18) Mahdavinia, G. R.; Hasanpour, J.; Rahmani, Z.; Karami, S.; Etemadi, H., Nanocomposite Hydrogel from Grafting of Acrylamide onto HPMC Using Sodium Montmorillonite Nanoclay and Removal of Crystal Violet Dye. Cellulose 2013, 20, 2591-2604.

(19) Ferfera-Harrar, H.; Aiouaz, N.; Dairi, N.; Hadj-Hamou, A. S., Preparation of Chitosan-gPoly(acrylamide)/Montmorillonite Superabsorbent Polymer Composites: Studies on Swelling, Thermal, and Antibacterial Properties. J. Appl. Polym. Sci. 2014, 131, 39747-39761.

(20) Gao, D. Y.; Heimann, R. B.; Williams, M. C.; Wardhaugh, L. T.; Muhammad, M., Rheological Properties of Poly(acrylamide)-Bentonite Composite Hydrogels. J. Mater. Sci. 1999, 34, 1543-1552.

(21) Slamova, R.; Trckova, M.; Vondruskova, H.; Zraly, Z.; Pavlik, I., Clay Minerals in Animal Nutrition. Appl. Clay Sci. 2011, 51, 395-398.

(22) Gao, G. R.; Du, G. L.; Sun, Y. N.; Fu, J., Self-Healable, Tough, and Ultrastretchable Nanocomposite Hydrogels Based on Reversible Polyacrylamide/Montmorillonite Adsorption. ACS Appl. Mater. Interfaces 2015, 7, 5029-5037.

(23) Zhao, X. H., Multi-Scale Multi-Mechanism Design of Tough Hydrogels: Building Dissipation into Stretchy Networks. Soft Matter 2014, 10, 672-687.

(24) Nakajima, T.; Kurokawa, T.; Ahmed, S.; Wu, W. L.; Gong, J. P., Characterization of Internal Fracture Process of Double Network Hydrogels under Uniaxial Elongation. Soft Matter 2013, 9, 1955-1966.

(25) Zhu, M. F.; Liu, Y.; Sun, B.; Zhang, W.; Liu, X. L.; Yu, H.; Zhang, Y.; Kuckling, D.; Adler, H. J. P., A Novel Highly Resilient Nanocomposite Hydrogel with Low Hysteresis and Ultrahigh Elongation. Macromol. Rapid Comm. 2006, 27, 1023-1028.

(26) Rinaudo, M., Chitin and Chitosan: Properties and Applications. Prog. Polym. Sci. 2006, $31,603-632$.

(27) Busilacchi, A.; Gigante, A.; Mattioli-Belmonte, M.; Manzotti, S.; Muzzarelli, R. A. A., Chitosan Stabilizes Platelet Growth Factors and Modulates Stem Cell Differentiation toward Tissue Regeneration. Carbohydr. Polym. 2013, 98, 665-676.

(28) Kumar, M.; Muzzarelli, R. A. A.; Muzzarelli, C.; Sashiwa, H.; Domb, A. J., Chitosan Chemistry and Pharmaceutical Perspectives. Chem. Rev. 2004, 104, 6017-6084.

(29) Rao, S. B.; Sharma, C. P., Use of Chitosan as a Biomaterial: Studies on Its Safety and Hemostatic Potential. J. Biomed. Mater. Res. 1997, 34, 21-28.

(30) Chen, B.; Evans, J. R. G.; Greenwell, H. C.; Boulet, P.; Coveney, P. V.; Bowden, A. A.; Whiting, A., A Critical Appraisal of Polymer-Clay Nanocomposites. Chem. Soc. Rev. 2008, 37, 568-594.

(31) Chen, B., Polymer-Clay Nanocomposites: An Overview with Emphasis on Interaction Mechanisms. Br. Ceram. Trans. 2004, 103, 241-249. 
(32) Wang, S. S.; Shu, Y. Q.; Liang, B. L.; Gao, L. C.; Gao, M.; Yin, P. G.; Guo, L., NacreInspired Green Artificial Bionanocomposite Films from the Layer-by-Layer Assembly of Montmorillonite and Chitosan. Chin. J. Polym. Sci. 2014, 32, 675-680.

(33) Shchipunov, Y.; Ivanova, N.; Silant'ev, V., Bionanocomposites Formed by in Situ Charged Chitosan with Clay. Green Chem. 2009, 11, 1758-1761.

(34) Al-Karawi, A. J. M.; Al-Qaisi, Z. H. J.; Abdullah, H. I.; Al-Mokaram, A. M. A.; AlHeetimi, D. T. A., Synthesis, Characterization of Acrylamide Grafted Chitosan and Its Use in Removal of Copper(II) Ions from Water. Carbohydr. Polym. 2011, 83, 495-500.

(35) Yazdani-Pedram, M.; Lagos, A.; Retuert, P. J., Study of the Effect of Reaction Variables on Grafting of Polyacrylamide onto Chitosan. Polym. Bull. 2002, 48, 93-98.

(36) Kinloch, Y., Fracture Behaviourof Polymers. Applied Science Publisher: London, 1983.

(37) Freier, T.; Koh, H. S.; Kazazian, K.; Shoichet, M. S., Controlling Cell Adhesion and Degradation of Chitosan Films by N-Acetylation. Biomaterials 2005, 26, 5872-5878.

(38) Tomihata, K.; Ikada, Y., In Vitro and in Vivo Degradation of Films of Chitin and Its Deacetylated Derivatives. Biomaterials 1997, 18, 567-575.

(39) Brouwer, J.; Vanleeuwenherberts, T.; Ottingvanderuit, M., Determination of Lysozyme in Serum, Urine, Cerebrospinal-Fluid and Feces by Enzyme-Immunoassay. Clin. Chim. Acta 1984, 142, 21-30.

(40) Porstmann, B.; Jung, K.; Schmechta, H.; Evers, U.; Pergande, M.; Porstmann, T.; Kramm, H. J.; Krause, H., Measurement of Lysozyme in Human-Body Fluids - Comparison of Various Enzyme-Immunoassay Techniques and Their Diagnostic Application. Clin. Biochem. 1989, 22, 349-355.

(41) Mahalingam, S.; Edirisinghe, M., Forming of Polymer Nanofibers by a Pressurised Gyration Process. Macromol. Rapid Comm. 2013, 34, 1134-1139.

(42) Chen, B.; Evans, J. R., Preferential Intercalation in Polymer-Clay Nanocomposites. J. Phys. Chem. B 2004, 108, 14986-14990.

(43) Fanood, M. H. R., Branching in Radical Polymerization of Acrylamide. Iran. Polym. J. 1998, 7, 59-65.

(44) Haraguchi, K.; Li, H.-J., Mechanical Properties and Structure of Polymer-Clay Nanocomposite Gels with High Clay Content. Macromolecules 2006, 39, 1898-1905.

(45) Zeng, M.; Feng, Z.; Huang, Y.; Liu, J.; Ren, J.; Xu, Q.; Fan, L., Chemical Structure and Remarkably Enhanced Mechanical Properties of Chitosan-graft-Poly (acrylic acid)/Polyacrylamide Double-Network Hydrogels. Polym. Bull. 2016, 1-20.

(46) Yu, L.; Ding, J. D., Injectable Hydrogels as Unique Biomedical Materials. Chem. Soc. Rev. 2008, 37, 1473-1481.

(47) Zhang, H. J.; Xia, H. S.; Zhao, Y., Poly(vinyl alcohol) Hydrogel Can Autonomously SelfHeal. ACS Macro Lett. 2012, 1, 1233-1236.

(48) Gleason, E. H.; Miller, M. L.; Sheats, G. F., A Radiotracer Study of Branch Formation in Polyacrylamide. J. Polym. Sci., Part A: Polym. Chem. 1959, 38, 133-139.

(49) Gough, K. M.; Zelinski, D.; Wiens, R.; Rak, M.; Dixon, I. M. C., Fourier Transform Infrared Evaluation of Microscopic Scarring in the Cardiomyopathic Heart: Effect of Chronic at(1) Suppression. Anal. Biochem. 2003, 316, 232-242.

(50) Mahdavinia, G. R.; Pourjavadi, A.; Hosseinzadeh, H.; Zohuriaan, M. J., Modified Chitosan 4. Superabsorbent Hydrogels from Poly(acrylic acid-co-acrylamide) grafted Chitosan with Saltand pH-Responsiveness Properties. Eur. Polym. J. 2004, 40, 1399-1407. 
(51) Mi, Y.; Xue, G.; Lu, X., A New Perspective of the Glass Transition of Polymer SingleChain Nanoglobules. Macromolecules 2003, 36, 7560-7566.

(52) Huang, Y.; Wang, D.; Zhu, X.; Yan, D.; Chen, R., Synthesis and Therapeutic Applications of Biocompatible or Biodegradable Hyperbranched Polymers. Polym. Chem. 2015, 6, 2794-2812. (53) Haraguchi, K., Synthesis and Properties of Soft Nanocomposite Materials with Novel Organic/Inorganic Network Structures. Polym. J. 2011, 43, 223-241.

(54) Liu, M.; Huang, J.; Luo, B.; Zhou, C., Tough and Highly Stretchable Polyacrylamide Nanocomposite Hydrogels with Chitin Nanocrystals. Int. J. Biol. Macromol. 2015, 78, 23-31.

(55) Okay, O.; Oppermann, W., Polyacrylamide-Clay Nanocomposite Hydrogels: Rheological and Light Scattering Characterization. Macromolecules 2007, 40, 3378-3387.

(56) Yao, H. B.; Tan, Z. H.; Fang, H. Y.; Yu, S. H., Artificial Nacre-Like Bionanocomposite Films from the Self-Assembly of Chitosan-Montmorillonite Hybrid Building Blocks. Angew. Chem. Int. Ed. 2010, 49, 10127-10131.

(57) Sun, J. Y.; Zhao, X. H.; Illeperuma, W. R. K.; Chaudhuri, O.; Oh, K. H.; Mooney, D. J.; Vlassak, J. J.; Suo, Z. G., Highly Stretchable and Tough Hydrogels. Nature 2012, 489, 133-136.

(58) Hu, Z. Q.; Chen, G. M., Novel Nanocomposite Hydrogels Consisting of Layered Double Hydroxide with Ultrahigh Tensibility and Hierarchical Porous Structure at Low Inorganic Content. Adv. Mater. 2014, 26, 5950-5956.

(59) Haraguchi, K., Nanocomposite Hydrogels. Curr. Opin. Solid St. M 2007, 11, 47-54.

(60) Sun, T. L.; Kurokawa, T.; Kuroda, S.; Bin Ihsan, A.; Akasaki, T.; Sato, K.; Haque, M. A.; Nakajima, T.; Gong, J. P., Physical Hydrogels Composed of Polyampholytes Demonstrate High Toughness and Viscoelasticity. Nat. Mater. 2013, 12, 932-937.

(61) Haraguchi, K.; Farnworth, R.; Ohbayashi, A.; Takehisa, T., Compositional Effects on Mechanical Properties of Nanocomposite Hydrogels Composed of Poly(N,N-dimethylacrylamide) and Clay. Macromolecules 2003, 36, 5732-5741.

(62) Xiong, L. J.; Hu, X. B.; Liu, X. X.; Tong, Z., Network Chain Density and Relaxation of in Situ Synthesized Polyacrylamide/Hectorite Clay Nanocomposite Hydrogels with Ultrahigh Tensibility. Polymer 2008, 49, 5064-5071.

(63) Thein-Han, W.; Kitiyanant, Y.; Misra, R., Chitosan as Scaffold Matrix for Tissue Engineering. Mater. Sci. Technol. 2008, 24, 1062-1075.

(64) Choi, B.; Loh, X. J.; Tan, A.; Loh, C. K.; Ye, E.; Joo, M. K.; Jeong, B., Introduction to in Situ Forming Hydrogels for Biomedical Applications. In In-Situ Gelling Polymers: For Biomedical Applications, Loh, J. X., Ed. Springer Singapore: Singapore, 2015, 5-35.

(65) Thian, E.; Huang, J.; Ahmad, Z.; Edirisinghe, M.; Jayasinghe, S.; Ireland, D.; Brooks, R.; Rushton, N.; Best, S.; Bonfield, W., Influence of Nanohydroxyapatite Patterns Deposited by Electrohydrodynamic Spraying on Osteoblast Response. J. Biomed. Mater. Res. A 2008, 85, 188194.

(66) White, S. R.; Sottos, N. R.; Geubelle, P. H.; Moore, J. S.; Kessler, M. R.; Sriram, S. R.; Brown, E. N.; Viswanathan, S., Autonomic Healing of Polymer Composites. Nature 2001, 409, 794-797.

(67) Ozawa, F.; Onoe, H.; Takeuchi, S. Highly Stretchable Cell-Laden Hydrogel Microfiber. In 18th International Conference on Miniaturized Systems for Chemistry and Life Sciences, MicroTAS 2014. Chemical and Biological Microsystems Society, 2014.

(68) Zhang, L.; Li, K. F.; Xiao, W. Q.; Zheng, L.; Xiao, Y. M.; Fan, H. S.; Zhang, X. D., Preparation of Collagen-Chondroitin Sulfate-Hyaluronic Acid Hybrid Hydrogel Scaffolds and Cell Compatibility in Vitro. Carbohydr. Polym. 2011, 84, 118-125. 
(69) Ionov, L., Hydrogel-Based Actuators: Possibilities and Limitations. Mater. Today 2014, 17, 494-503. 